$R M x A C, \mathbf{5 3}, 190-197$ (2021)

(c) 2021: Instituto de Astronomía, Universidad Nacional Autónoma de México

https://doi.org/10.22201/ia.14052059p.2021.53.38

\title{
CHARACTERIZATION OF MODERN CCD AND CMOS SENSORS FOR SKY SURVEYS
}

\author{
S. Karpov ${ }^{1}$, A. Christov ${ }^{1}$, A. Bajat ${ }^{1}$, R. Cunniffe ${ }^{1}$, and M. Prouza ${ }^{1}$
}

\section{RESUMEN}

Se muestra el trabajo realizado en un laboratorio recientemente establecido dentro del Instituto de Física en Praga para caracterizar los sensores modernos CCD y CMOS de gran formato para aplicaciones de cartografiado del cielo. Mientras el laboratorio se estableció principalmente para participar en la caracterización de sensores CCD de bajo nivel para el proyecto LSST, también pudimos realizar una prueba exhaustiva de laboratorio de cámaras sCMOS (como la Andor Marana) que es especialmente interesante para aplicaciones de cartografiado de grandes regiones del cielo amplio, debido a su gran formato, diseño retroiluminado, alta velocidad de toma de imágenes y bajo ruido de lectura). También se realizaron mediciones detalladas de no linealidad de respuesta de cámaras CCD de Moravian Instruments como el modelo G4-16000 (basado en el chip Kodak KAF-16803 de gran formato) que es utilizado en varios telescopios robóticos. Se revisan los resultados adquiridos por estas cámaras, así como el hardware y software que desarrollamos para el laboratorio.

\section{ABSTRACT}

Here we review the efforts we take in a newly established laboratory inside Institute of Physics in Prague in order to characterize modern large-format CCD and CMOS sensors for sky survey applications. While the laboratory is primarily established in order to participate in low-level CCD sensor characterization for LSST project, we also managed to perform a thorough laboratory testing of recently released Andor Marana sCMOS (which is especially interesting for wide-field sky monitoring applications due to its large format, back-illuminated design, high achievable frame rate and low read-out noise), as well as detailed measurements of response non-linearity of Moravian Instruments G4-16000 CCD cameras (based on large-format Kodak KAF-16803 chip) used in several robotic telescopes. We briefly review the results acquired on these cameras, as well as hardware and software we developed for the laboratory.

Key Words: detectors — instrumentation: miscellaneous

\section{INTRODUCTION}

Sky survey applications require large format image sensors with high quantum efficiency, low readout noise, fast read-out and a good inter- and crosspixel stability and linearity. Charge-Coupled Devices (CCDs), typically employed for such tasks, lack only the read-out speeds, which significantly lowers their performance for detecting and characterizing rapidly varying or moving objects. On the other hand, recent development in the low-noise Complementary Metal-Oxide-Semiconductor (CMOS) architectures (see e.g. Vu et al. (2008)) allowed to design and create a market-ready large-format CMOS chips with read-out noise as low as 1-2 electrons, on par with best CCDs (Fowler et al. 2010) - socalled "scientific CMOS" (sCMOS) chips. Like standard CMOS sensors (and unlike CCDs), they did not perform any charge transfer between adjacent pixels, employing instead individual column-level ampli-

\footnotetext{
${ }^{1}$ CEICO, Institute of Physics, Czech Academy of Sciences, Prague, Czech Republic.
}

fiers with parallel read-out and dual 11-bit analog-todigital converters (ADCs) operating in low-gain and high-gain mode, correspondingly, and an on-board field-programmable gate array (FPGA) logic scheme that reconstructs a traditional 16-bit reading for every pixel from two 11-bit ones.

Complexities of such devices require thorough understanding and characterization in order to utilize their complete potential in scientific applications requiring high precision - e.g. modern large-scale sky survey applications. To facilitate such characterization of both CCD, sCMOS, and potentially other promising kinds of large format imaging detectors, we established the dedicated laboratory inside Institute of Physics in Prague. In doing so, we tried as much as possible to re-use already acquired experience of operating similar systems gathered by our collaborators within the Large Synoptic Survey Telescope (LSST) (Doherty et al. 2014; Kotov et al. 2016; Weatherill et al. 2017) and other projects as well. 

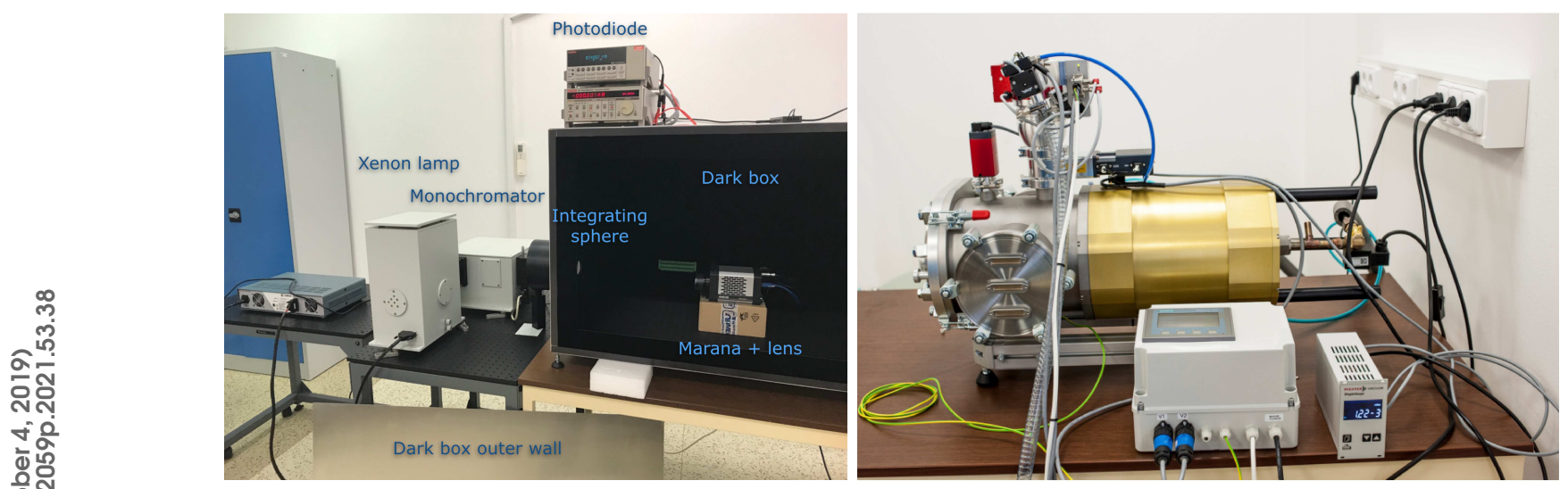

Fig. 1. Left panel - laboratory setup at Institute of Physics, Czech Academy of Sciences, as used for testing Andor Marana sCMOS camera in Mar 2019. The side wall of a dark box is open so the camera inside it is visible. The photodiode used to control light source intensity is installed in a side port of the integrating sphere, and is measured by a dedicated picoammeter. Right panel - the vacuum chamber attached to the dewar (gold-colored cylinder). The pumps assembly and the pressure sensor are mounted on the top. In front of the assembly are the Siemens PLC and the pressure readout unit.

The laboratory setup is described in Section 2, and some of our activities regarding characterization of both CCD and CMOS detectors - in Sections 3 and 4 , correspondingly.

\section{LABORATORY SETUP}

Our laboratory setup (see Figure 1) contains most of facilities expected from an optical sensor testbench, including a cryogenic system, a "dark box", light source and monochromator, and a set of sensors, all wired to a dedicated laboratory server powered by a software package specifically designed by us to operate the laboratory.

The main parts of the vacuum and cryogenic system are the dewar and the vacuum chamber, shown in right panel of Figure 1. The dewar has an inner container to hold liquid nitrogen (LN), serving as a coolant, and has an 8 inch cold plate on the vacuum chamber side. The LN is pumped in through an inlet at the back of the dewar, which also serves as a vent for the gaseous nitrogen. A custom made vacuum chamber is mounted to the front of the dewar, forming a common volume. This volume is evacuated to a high vacuum to ensure thermal isolation of the cold masses, prevent water condensation and contamination of the detectors being tested. The chamber is made of stainless steel with two flanges on each side and quick release door at the front, equipped with an exchangeable glass window made of an UV-grade fused silica with $88 \%$ transitivity at $185 \mathrm{~nm}$. Each of the side flanges has three 50-pin feedthroughs for the detector readout/control or auxiliary connections.

We use a sequence of two pumps, a membrane one and a turbo-molecular one for achieving a high vac- uum. The pumps and the valves are controlled by a Siemens PLC implementing the operation sequences. A cryogenic temperature controller, CryoCon $24 \mathrm{c}$, is used to actively control the temperature by providing current for heaters mounted on the cold mass.

Directly in front of the vacuum chamber is a "dark box" - a light tight chamber $1.2 \mathrm{~m}$ long and $60 \mathrm{~cm}$ wide and tall, intended to allow the light to propagate for some distance from the source (integrating sphere) before arriving at the sensor and therefore to improve the illumination uniformity. A secondary purpose of the dark box is to have space for installing additional optical components, e.g. a system to project patterns onto the tested sensor in the vacuum chamber. The "dark box" may also be used for testing a cameras what do not have to be in the cold chamber, e.g. thermo-electrically cooled ones.

The light source is a Camlin ALTAS 200 monochromator powered by an APOLLO X-600 Xenon lamp and equipped with manual entrance and exit slits, and two (optical and near-infrared), feeding an integrating sphere through a fiber bundle. The integrating sphere is equipped with an intensity monitoring light sensor, and is mounted directly onto the side of the dark box.

The whole system is controlled by a dedicated CCDLAB software (Karpov \& Christov 2018), which performs real-time monitoring of a system state and stores it to a database for analyzing its evolution, displays it in a user-friendly web interface, and also allows easy scripting control of its operation. 

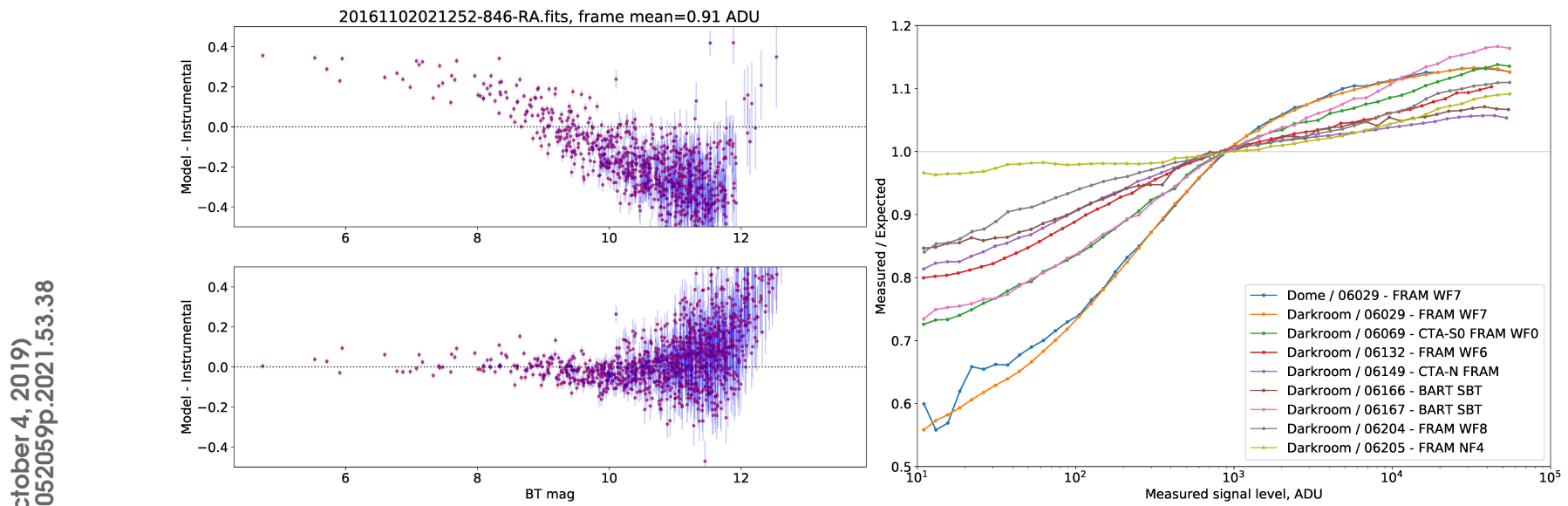

Fig. 2. Non-linearity of Moravian Instruments G4-16000 cameras. Left panel - photometric residuals after fitting the instrumental magnitudes measured on a frame with low mean intensity level with a model including catalogue magnitudes, color term to account for a difference in photometric system, and low-order spatial polynomial to fix residual uncorrected vignetting. Upper panel - original uncorrected data, lower panel - the data corrected for detector response non-linearity. Outliers there correspond mostly to blended stars where aperture photometry we use performs badly, as well as to intrinsic photometric problems of the catalogue and a difference of photometric systems. Right panel - results of non-linearity measurement for a number of cameras based on the same chip model, mostly measured on a dedicated calibration stand in a dark room conditions. There is a tentative decrease of the level of non-linearity with the increase of camera serial number, what may correspond to the gradual improvements of the manufacturing process and electronics used in the cameras.

\section{NON-LINEARITY CHARACTERIZATION OF MORAVIAN INSTRUMENTS G4-16000 CAMERAS}

Moravian Instruments G4-16000 are an inexpensive large-format CCD cameras based on Kodak KAF-16803 chip and widely used in several European amateur and professional astronomical projects, including FRAM telescopes (Prouza et al. 2010; Janeček et al. 2017). In order to achieve the best possible photometric accuracy for them, we initiated a thorough study of detector response nonlinearity (see left panel of Figure 2 for an example of this effect manifestation in photometric calibration) of these cameras, and developed a dedicated measurement routine, applicable both in the laboratory and on an actual remote telescope setup.

Both experiments were performed according to the same protocol, which consisted of acquiring a number of images with different exposures and then studying in the dependence of signal level on exposure time. To do so, we acquired a series of "light" exposures, with shutter fully open, each immediately followed by a "dark" exposure of the same length with closed shutter to control the bias level of the images. To exclude any possible bias related to slow changes in the intensity of a light source, parameters of environment and camera electronics, we randomized the sequence of exposures, so that on every step we randomly sampled the exposure from log- uniform distribution between $0.1 \mathrm{~s}$ and $300 \mathrm{~s}$. Moreover, to have a more explicit control on the stability of the screen illumination by light source, after every "light+dark" image pair with a random exposure, we acquired a similar pair of "control" images with an exposure arbitrarily fixed to $10 \mathrm{~s}$.

In the laboratory, we performed a number of experiment runs with different amounts of light reaching the detector. In the telescope experiment setup, the intensity of light was constantly changing due to day/night cycle. Every run contained several hundreds to thousands of images acquired over long time intervals, from several hours up to several days.

To speed up the frame read-out, we acquired only the central $1024 \times 1024$ pixels region of every image, which resulted in a 6 seconds read-out time. Then we subtracted the bias pattern from every "light" image, isolated the regions of incoming gradient image with similar intensities, and computed the mean signal values over these regions to be used in the analysis.

We define the detector non-linearity $N$ as a ratio between the observed light fluence (measured signal level) $I_{\mathrm{obs}}$ and the "expected" value directly proportional to the exposure time $T_{\exp }$ :

$$
N=I_{\mathrm{obs}} /\left(F \cdot T_{\exp }\right) .
$$

where $F$ is the scaling coefficient corresponding to the incoming light flux level. In all our setups 
the intensity of illumination varied slowly over time, either due to the variations of the light source parameters with temperature in calibration stand setup, or due to changes of the in-dome illumination related to the motion of the Sun and clouds in on-telescope setup. Therefore we reconstructed the per-frame values of $F$ using the recorded "control" images all having constant exposure of 10 seconds as:

$$
F \propto I_{\mathrm{obs}, 10} / N\left(I_{\mathrm{obs}, 10}\right)
$$

where $I_{\mathrm{obs}, 10}$ may be interpolated on any given moment, assuming smooth variations of incoming light, and $N$ term accommodates for the fact that "calibration" frames are also affected by the detector response non-linearity. The scaling coefficient here may be chosen depending on the shape of empirical dependence of the signal level on exposure. For our analysis, we quite arbitrarily defined it in a such way that the mean non-linearity is equal to unity over the interval of intensities between 300 and 3000 ADU.

Finally, we may iteratively solve Eq. 1 together with Eq. 2 to reconstruct the detector non-linearity as a function of measured signal level.

Our experiments demonstrated that both experiment setups deliver the same non-linearity curve, perfectly stable for a given camera, and showing a significantly non-linear response (see right panel of Figure 2 for the results for all cameras we tested), with measured signal level nowhere directly proportional to the exposure time (which would manifest as a horizontal segment on the plot). Instead, the nonlinearity curve shows a characteristic shape consisting of several (two or maybe even three) log-linear segments with different slopes, with non-linearity being the most significant in the low intensity region. The response is also non-linear in the high intensity region, approximately above half of dynamic range $\left(\sim 30000\right.$ ADUs, or $\left.\sim 48000 \mathrm{e}^{-}\right)$- the behaviour not uncommon for a typical CCDs.

The shape of non-linearity curves is similar among all cameras we tested, with a tentative improvement towards the ones manufactured more recently, i.e. having greater serial numbers. They all show a characteristic piece-wise structure with a slope break at around $1000 \mathrm{ADU}$, which may quite easily be fitted by two separate log-linear segments of the form:

$$
N(I)=p_{0} \cdot \lg I+p_{1}+\mathcal{H}\left(\lg I-B_{1}\right) \cdot\left(p_{2} \cdot \lg I+p_{3}\right)
$$

where $\mathcal{H}(x)$ is a Heaviside step function, and the $p_{i}$ and $B_{1}$ parameters are fitted for every chip.

The signal on the detector may be linearized by dividing the observed bias and dark subtracted value with $N$ as:

$$
I_{\text {linear }}=I_{\text {obs }} / N\left(I_{\text {obs }}\right)
$$

Lower left panel of Figure 2 shows the effect of a linearization of the data on photometric residuals.

\section{CHARACTERIZATION OF ANDOR MARANA SCMOS CAMERA}

Andor Marana (Andor 2019) is a novel camera built around back-illuminated GSense400BSI sCMOS chip. We decided to perform its laboratory and on-sky characterization using the camera generously provided to us by manufacturer for testing, in order to assess its performance for sky survey applications. Such a device, if proven to be stable enough, may be of extreme importance for a tasks of precise photometry in wide field sky surveys, especially when high temporal resolution is desirable, e.g. for the detection and study of rapid optical transients (Karpov et al. 2010, 2019a), space debris tracking (Karpov et al. 2016) or observations of faint meteors (Karpov et al. 2019b). Due to large frame format, absence of microlens raster on top of the chip, good quantum efficiency and fast read-out, such device may also be a promising detector for a next generation of FRAM atmospheric monitoring telescopes (Prouza et al. 2010; Janeček et al. 2017).

For the camera testing we used a subset of equipment of our laboratory in a setup shown in left panel of Figure 1. That included a fully light-isolating dark box, monochromator with Xenon lamp, and an integrating sphere mounted directly on the input port of a dark box. A dedicated photodiode coupled with Keithley picoammeter was used to control the intensity of light inside the integrating sphere. The camera was controlled by a dedicated FAST data acquisition software (Karpov 2018) specifically designed for operating fast frame rate scientific cameras of various types and integrated into the CCDLAB software which provided overall control and monitoring of the whole laboratory setup.

To protect the camera from dust, we equipped it with a Nikkor 300 f/2.8 lens, adjusted in such way as to provide illumination of the whole chip with the light from integrating sphere output window. Due to lens vignetting, this resulted in a slightly bellshaped flat fields. The same lens has been later used for the on-sky testing of the camera photometric performance. For it, the camera with lens were installed on a Software Bisque' Paramount ME mount, controlled with RTS2 software (Kubánek et al. 2004). The field of view of the camera in this setup was $4.26^{\circ} \times 4.26^{\circ}$ with $7.5^{\prime \prime} /$ pixel scale. The whole setup was installed in the dome of FRAM telescope being 

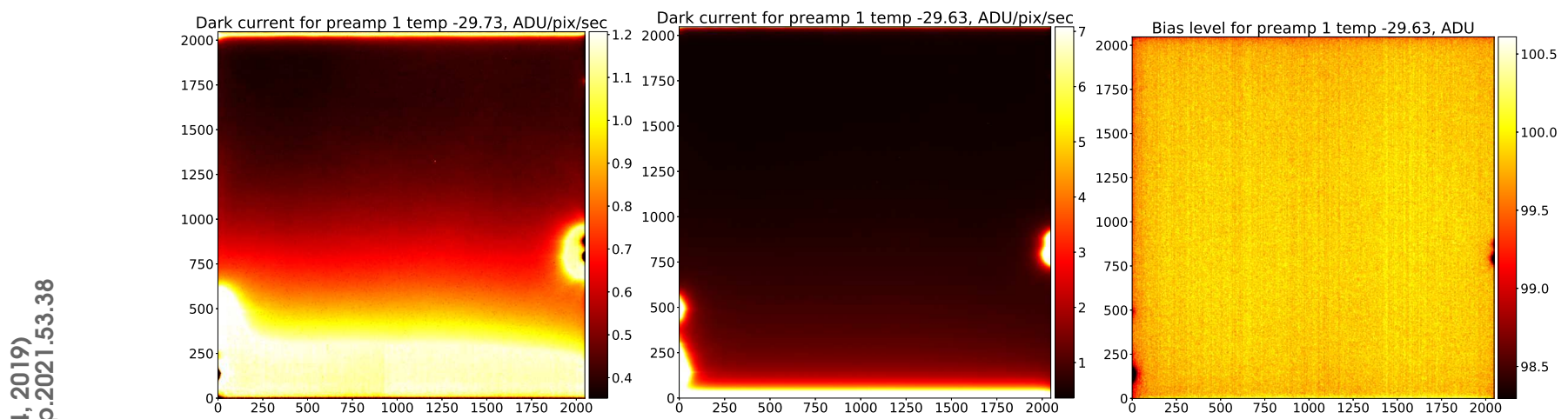

Fig. 3. Maps of a dark current for a default camera regime with Anti-Glow correction (left, median value is 0.55 $\mathrm{ADU} / \mathrm{pixel} / \mathrm{s}$ ) and with correction disabled (middle, median value is $0.84 \mathrm{ADU} / \mathrm{pixel} / \mathrm{s}$ ), as well as of bias level (right).

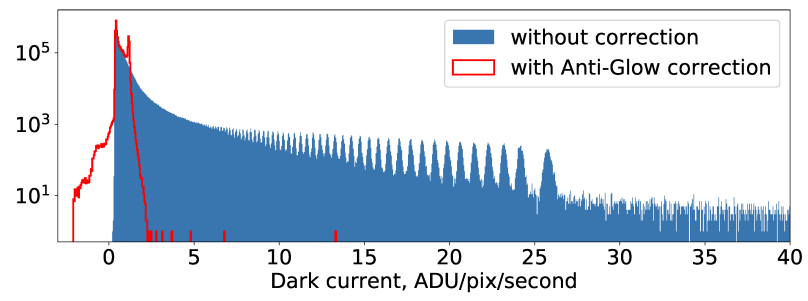

Fig. 4. Histograms of a per-pixel dark current in default camera regime (with Anti-Glow correction) and with correction disabled.

tested and commissioned at the same time at the backyard of Institute of Physics of Czech Academy of Sciences in Prague.

The laboratory testing consisted by a scripted set of imaging sequences of various exposures and durations, acquired under different light intensities or in the dark, and with different readout settings. Most of the sequences were acquired with chip temperature set to $-30^{\circ} \mathrm{C}$, which the camera' thermo-electric cooler was able to continuously support within the closed area of the dark box with just an air cooling during the whole duration of our experiments. However, for some tests the temperature was also varied. The majority of tests have been performed with a high gain (16-bit) regime, as a most convenient for astronomical applications.

Over every sequence, pixel-level mean values ("mean frames") and standard deviations ("standard deviation frames") have been constructed; over some of the sequences, pixel values on individual frames have also been specifically studied.

\subsection{Dark current}

Figures 3 and 4 shows the maps and histograms of camera dark current, derived by acquiring a sets of dark frames with varying exposure times, for a default operation with an "Anti-Glow technology" onboard correction algorithms turned on, and with the latters disabled. It may be seen that these algorithms over-correct the dark current for a limited $(\propto 0.15 \%)$ set of pixels, rendering their dark current formally negative. Turning off these corrections removes such negative values from the dark current map and significantly increased the amplitude of edge glow spots, leading to a long tail in the histogram of its values, and slightly increasing the dark current median value (from $0.55 \mathrm{ADU} / \mathrm{pixel} / \mathrm{s}$ to $0.84 \mathrm{ADU} / \mathrm{pixel} / \mathrm{s}$ ).

The linear slope of over-compensated pixels suggest that the Anti-Glow correction is a linear subtraction of a some pre-computed map multiplied by exposure time, and is therefore equivalent to standard astronomical procedure of dark current correction. Therefore, we suggest disabling this correction when using the camera in a properly calibrated environment in order to avoid possible problems due to over-compensation.

\subsection{Photon transfer curve and linearity}

In order to build the photon transfer curve (PTC, the dependence of pixel RMS value on its mean, see Janesick (2007)), we acquired a series of frames with varying exposure time during constant illumination. Due to slightly different properties of individual pixel circuits, column level amplifiers and ADCs, we expect the properties to vary on a pixel to pixel basis, therefore we did not perform any spatial averaging, using instead just a temporal mean and variance of every pixel readings. The resulting PTC is shown in Figure 5, along with the effective gain computed from it. The sharp jump is seen around 1500 ADU in both plots there, corresponding to the transition between low-gain and high-gain amplifiers. The gain below the transition nicely corresponds to the one reported by manufacturer; above 

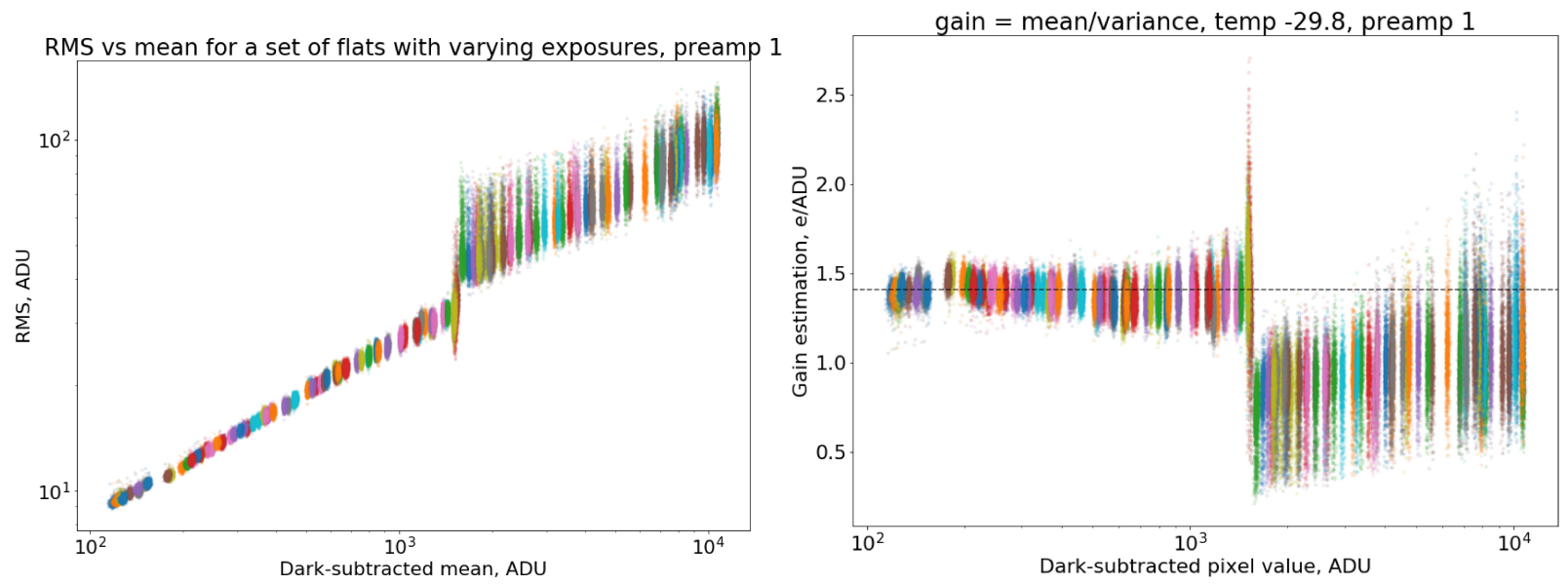

Fig. 5. Left panel - photon transfer curve, i.e. dependence of a pixel temporal variance on (dark level subtracted) mean value. Right panel - gain estimated from photon transfer curve as a function of pixel value. On both panels, different colors represent different sequences of frames with varying exposures, and the spread of points of same color - the difference of corresponding values across different pixels of the sensor. Dashed horizontal line on right panel represents the manufacturer-provided gain level of $1.41 \mathrm{e}^{-} / \mathrm{ADU}$. The jump at around 1500 ADU represents the transition between low-gain and high-gain amplifiers, providing different effective gain and having different spatial structures.

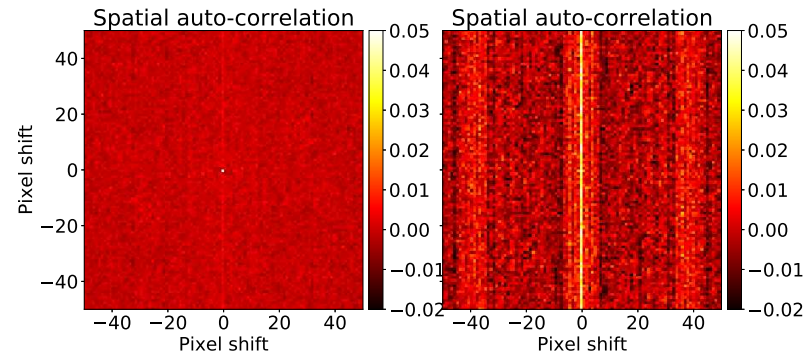

Fig. 6. Mean spatial autocorrelation for the gain maps computed for mean values between 500 and 1000 ADU (left panel) and between 2500 and 5000 ADU (right panel). The latter shows a distinct column-like structure, reflecting different effective gains for different column level amplifiers.

the transition, the effective gain drops by nearly two times. Also, the spatial scatter of gain values across the sensor changes - from nearly uniform below the transition to a significantly varying ones above it. Spatial auto-correlation shown in Figure 6 confirms it, also suggesting that the scatter actually reflects a bit different gain settings for different column level amplifiers.

Linearity curve shown in Figure 7, on the other hand, does not show any significant jump of comparable amplitude at the transition region, with the slight discontinuity there on the level typically below $2 \%$, which has a character of a small multiplicative coefficient. Above half of dynamic range, however, there is a systematic change of linearity slope, which may reach up to $10 \%$ towards the saturation point.

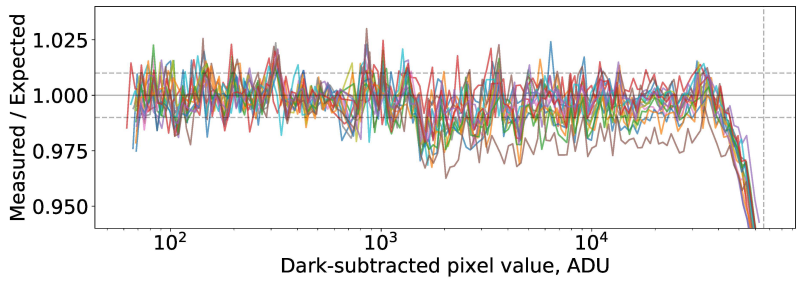

Fig. 7. Linearity curve for a random set of pixels. The curve represent the ratio of an actually measured signal to the one expected for a linear signal scaling with exposure time, with interval below 1000 ADU used to define a linear slope. Dashed vertical line to the right marks the position of a digital saturation (65535 ADU), systematic significant deviations from linearity start at about half of this value. The amplifier transition region is easily visible at around $1500 \mathrm{ADU}$, with the jump amplitude of typically less than couple of percents.

Overall, the linearity of Marana camera seems a bit better than the one seen in older Andor Neo, where the slope of linearity curve changed above the transition region, with the non-linearity reaching up to 5\% (Karpov et al. 2019a).

The transition region itself also shows an additionally increased RMS level due to sporadic change between the readings of both amplifiers, as shown in right panel of Figure 8 .

This all suggests that the properties of flat fields should be a bit different when acquired at intensities below and above the amplifier transition (i.e. 1500 $\mathrm{ADU})$, and requires a more detailed study if one is 

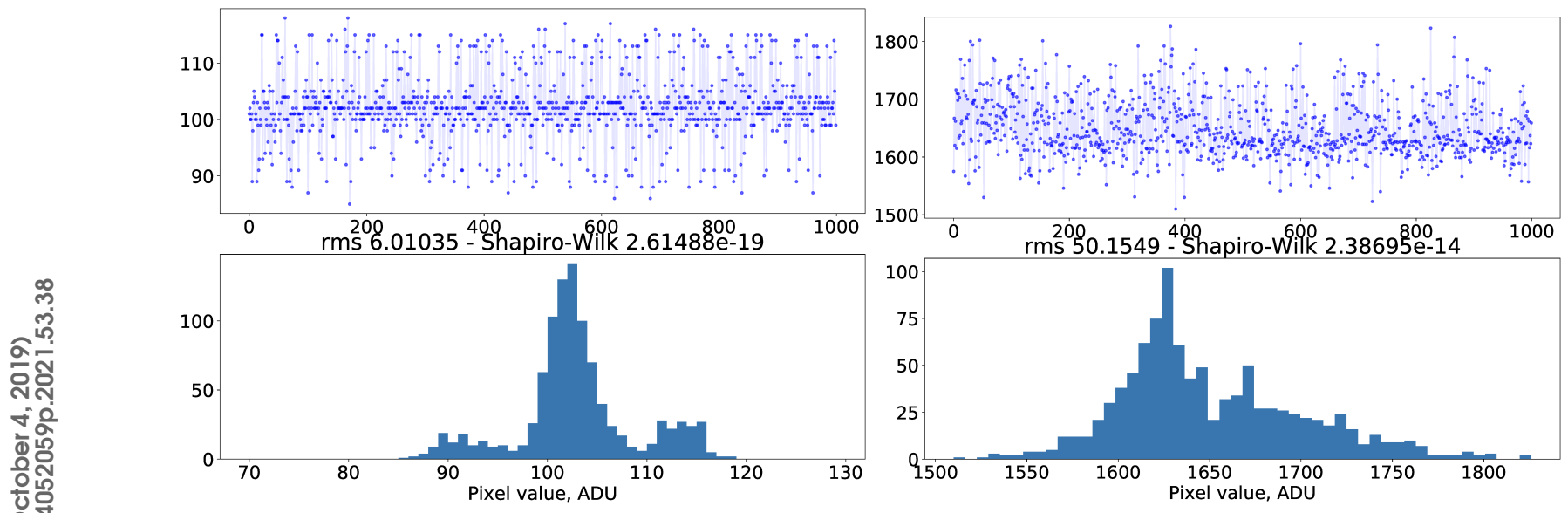

Fig. 8. Examples of noise sources specific for a sCMOS architecture. Left panel - noisy pixel on a dark frame that displays distinctive Random Telegraph Signal (RTS) switching between several bias states, showing temporal sequence of pixel values, as well as histogram of these values. Right panel - the same for a pixel at the amplifier transition intensity region. Excessive noise is caused by the "jumping" between the readings of high gain and low gain amplifiers.

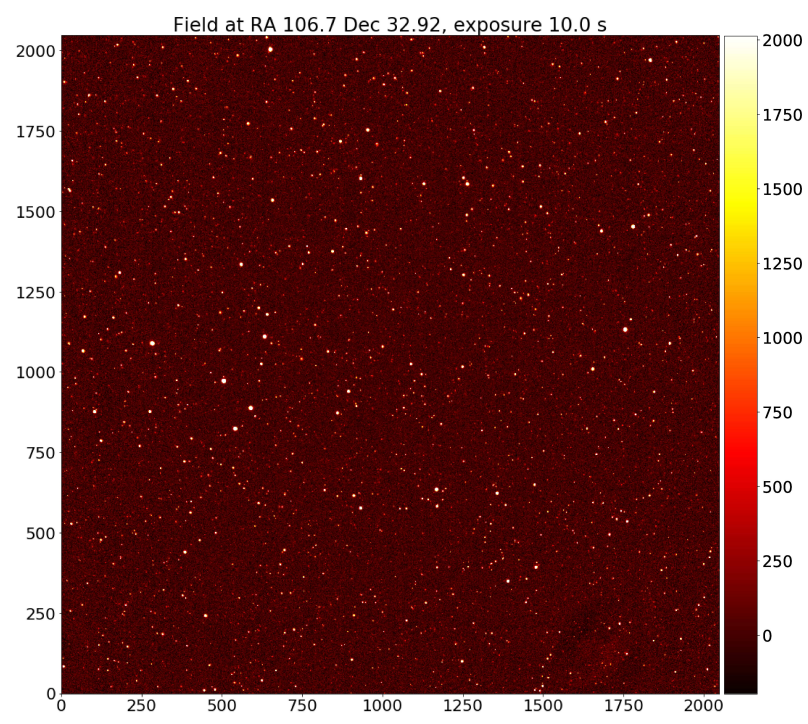

Fig. 9. Single frame from on-sky testing of Andor Marana sCMOS, equipped with Nikkor $300 \mathrm{f} / 2.8$ lens with no color filters. The frame is dark subtracted and flat-fielded using evening sky flat. The field of view is $4.26^{\circ} \times 4.26^{\circ}$ with $7.5^{\prime \prime}$ / pixel scale. Median FWHM of the stars is 2.1 pixels, making the image nearly critically sampled. Note the absence of cosmetic defects typical for CCD frames - hot and dark columns, bleedings from oversaturated stars, etc.

aimed at precise photometric applications at different intensity levels.

\subsection{On-sky testing}

On-sky testing of the camera consisted of a series of continuous observations of a fixed sky positions in order to assess the photometric performance and achievable stability of the data. Every frame (example is shown in Figure 9) acquired in such regime was bias and dark subtracted and normalized to a flat field acquired by averaging evening sky images. Then every frame was astrometrically calibrated using Astrometry.Net (Lang et al. 2010) code, and star detection and measurements were performed using routines available in SEP (Barbary 2018) Python package, based on original SEXTRACTOR code by Bertin \& Arnouts (1996). On every frame, the zero point model was constructed by cross-matching the object list with the synthetic photometric catalogue of Pickles \& Depagne (2010) and fitting their instrumental magnitudes with a catalogue $V$ values as a base, $J-K$ as a color term, and a third order spatial polynomial to compensate imperfections of evening flats, as well as positional-dependent aperture correction due to changes of stellar PSF. By comparing the fits from different frames, the color equation for these unfiltered observations was found to be:

$$
\text { Instr }=V-0.49 \cdot(J-K) .
$$

After that, the color term was kept fixed for all frames, and the fitting was performed only for spatial polynomial part. All photometric measurements of all stars on all frames were then positionally clustered and separated into light curves corresponding to individual objects. Then for every light curve the mean value and standard deviation were computed. The scatter versus magnitude plot for the lightcurves is shown in Figure 10, and demonstrates that the photometric precision of measurements with this sensor easily reaches $1 \%$ in the setup we used. We did not detect any systematic effects dependent 


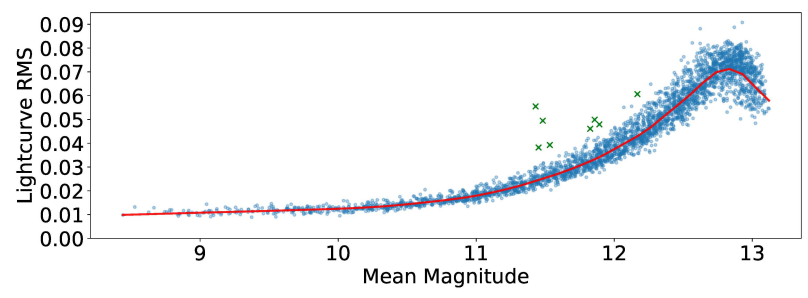

Fig. 10. The scatter of photometric measurements of individual star along the sequence of sky images versus its mean value. A few outliers are caused by errorneous measurements of a blended stars.

on sub-pixel position on the level greater $0.5 \%$, which is consistent with the chip being back-illuminated.

\section{CONCLUSIONS}

The laboratory for sensor and camera testing at the Institute of Physics (FZU) of the Czech academy of sciences is still under heavy development. We plan, in addition to the setup described above, to include a $\mathrm{Fe}^{55} \mathrm{X}$-ray source, pattern projection capabilities and a tungsten lamp, which will allow for a more thorough and detailed detector characterization (e.g. studying gain stability, sub-pixel sensitivity variations, etc).

We already performed laboratory study of a set of cameras based on large-format CCD and CMOS chips. It allowed us to characterize the non-linearity of G4-16000 cameras and to construct an instrument signature removal (ISR) routine for them, which is an essential step in creating precise photometric pipeline for these cameras used on a FRAM telescopes.

We also performed a thorough laboratory and on-sky testing of a recently released Andor Marana sCMOS camera, and demonstrated that it is indeed a very promising camera for a sky survey applications, especially requiring high temporal resolution.

Acknowledgments: This work was supported by European Structural and Investment Fund and the Czech Ministry of Education, Youth and Sports
(Project CoGraDS - CZ.02.1.01/0.0/0.0/15 003/0000437). Authors are grateful to Andor, Oxford Instruments Company for providing the camera used for testing.

\section{REFERENCES}

Andor, O. I. C. 2019, Marana sCMOS Specifications, published online at https://andor.oxinst.com/ assets/uploads/products/andor/documents/ Marana-sCMOS-Specifications.pdf

Barbary, K. 2018, SEP: Source Extraction and Photometry

Bertin, E. \& Arnouts, S. 1996, A\&AS, 117, 393

Doherty, P. E. et al. 2014, SPIE, 9154, 9154

Fowler, B., Liu, C., Mims, S., et al. 2010, SPIE, 7536, 07

Janesick, J. R. 2007, Photon Transfer DN $\rightarrow \lambda$

Janeček, P., Ebr, J., Blažek, J., et al. 2017, EPJW, 144, id.01012

Karpov, S. 2018, FAST data acquisition software, published online at https://github.com/karpov-sv/ fast

Karpov, S., Beskin, G., Biryukov, A., et al. 2019a, RMxAC, 51, 30

Karpov, S., Beskin, G., Bondar, S., et al. 2010, AdAst, 2010, 784141

Karpov, S. \& Christov, A. 2018, CCDLab laboratory control software, published online at https://github. com/karpov-sv/ccdlab

Karpov, S., Cunniffe, R., Ebr, J., \& Janeček, P. 2018, AN, 339, 391

Karpov, S., Katkova, E., Beskin, G., et al. 2016, RMxAC, 48, 112

Karpov, S., Orekhova, N., Beskin, G., et al. 2019b, RMxAC, 51, 127

Kotov, I. V., Haupt, J., O'Connor, P., et al. 2016, SPIE, 9915, 9915

Kubánek, P., Jelínek, M., Nekola, M., et al. 2004, AIPC, 727,753

Lang, D., Hogg, D. W., Mierle, K., Blanton, M., \& Roweis, S. 2010, AJ, 139, 1782

Pickles, A. \& Depagne, É. 2010, PASP, 122, 1437

Prouza, M., Jelínek, M., Kubánek, P., et al. 2010, AdAst, 2010, 849382

Vu, P., Fowler, B., Liu, C., et al. 2008, SPIE, 7021, 03

Weatherill, D., Arndt, K., Plackett, R., \& Shipsey, I. 2017, JInst, 12, C12019 